\title{
Overexpression of VEGF and TGF-B1 mRNA in Pap smears correlates with progression of cervical intraepithelial neoplasia to cancer: Implication of YY1 in cervical tumorigenesis and HPV infection
}

\author{
STAVROULA BARITAKI ${ }^{1,2}$, STAVROS SIFAKIS ${ }^{3 *}$, SARA HUERTA-YEPEZ ${ }^{4 *}$, IOANNIS K. NEONAKIS ${ }^{5}$, \\ GIANNOULA SOUFLA $^{1}$, BENJAMIN BONAVIDA ${ }^{2}$ and DEMETRIOS A. SPANDIDOS ${ }^{1}$
}

\author{
${ }^{1}$ Department of Virology, Medical School, University of Crete, Heraklion, Crete, Greece; ${ }^{2}$ Department of Microbiology, \\ Immunology and Molecular Genetics, David Geffen School of Medicine, Johnson Comprehensive Cancer Center, \\ University of California - Los Angeles, CA, USA; ${ }^{3}$ Department of Obstetrics and Gynaecology, University Hospital \\ of Crete, Heraklion, Crete, Greece; ${ }^{4}$ Hospital de Infectologia, CMN 'La Raza' UIM en Infectologia e Immunologia, \\ Mexico City, Mexico; ${ }^{5}$ Mycobacteriology Laboratory, University Hospital of Crete, Heraklion, Crete, Greece
}

Received April 2, 2007; Accepted May 3, 2007

\begin{abstract}
The screening of neo-angiogenesis related gene expression has uncovered many disrupted molecular pathways which may significantly confer to malignant transformation of various cell types including cervical cells. The objective of the present study was to delineate whether changes in certain gene expression profiles during the malignant conversion of the uterine cervix can be potentially used to predict the clinical course and outcome of the cervical pathology. Total RNA was isolated from Pap smears obtained from healthy females or patients diagnosed with low-grade squamous cervical intraepithelial lesions (LG-SIL), high-grade (HG)-SIL or cervical carcinoma. VEGF, TGF-ß1 and YY1 mRNA expression levels were assessed by QRT-PCR. Confirmation of YY1 protein discrepancy among cervical tissues of different histopathology was performed by immunohistochemistry. All tested genes showed statistically significant expression variations among the indicated groups. VEGF and TGF- 11 mRNA overexpression was found to be associated with progression from low-grade to high-grade cervical intraepithelial neoplasia (CIN), while YY1 showed constitutively elevated transcript levels in CIN
\end{abstract}

Correspondence to: Professor D.A. Spandidos, Laboratory of Virology, Medical School, University of Crete, P.O. Box 1527, Heraklion 710 03, Crete, Greece

E-mail: spandidos@spandidos.gr

${ }^{*}$ Contributed equally

Key words: uterine cervical cancer, cervical intraepithelial neoplasia, real-time PCR, vascular endothelial growth factor, transforming growth factor- $\beta 1$, Yin-Yang 1 and cervical cancer compared to controls. At the protein level YY1 was also overexpressed in HG-SIL and cancer tissues compared to LG-SIL. Both YY1 transcript and protein overexpression were associated with HPV18- or HPV16-infected samples. Spearman analysis revealed a co-expression pattern for VEGF and TGF-ß1 mRNAs in normal cervix and LG-SIL; however, YY1 expression correlated negatively with VEGF and TGF- $\beta 1$ transcript levels upon the onset of the cervical neoplastic transformation. Our findings provide for the first time evidence for the implication of YY1 in uterine cervix carcinogenesis and suggest that VEGF, TGF- $\$ 1$ and YY1 could be useful biomarkers of cervical malignant transformation as well as potential targets for therapeutic approaches.

\section{Introduction}

The malignant transformation of the uterine cervix represents an excellent paradigm of disrupted expression of growth factors (GFs) at different levels, following the onset of an initial human papilloma virus (HPV) infection, the progression to CIN and finally to cancer $(1,2)$. Despite the fact that the chronic infection of keratinocytes of the uterine cervix by HPV is thought to be strongly implicated as a causative agent in the etiology of cervical cancer and its precursors (CIN), HPV infection alone could not be considered sufficient for cancer development (3). Thus, a number of exogenous and abnormally expressed endogenous factors, including transcriptional regulators, may be conducive to CIN and tumor advancement by acting independently or synergistically. Among the various angiogenic factors that have been studied in tissues derived from patients with cervical lesions and cancer, TGF- $\$ 1$ and VEGF have shown significant disrupted expression at different levels, even in early-stage cervical abnormalities (LG-SIL), compared to normal tissues $(1,4-8)$.

TGF- $\beta$ represents a family of peptides of which TGF- $\beta 1$, -2 , and -3 are expressed in humans (9). These cytokines have 
been shown to be produced by various cell types, including keratinocytes and cells implicated in immune responses much like $\mathrm{T}$ lymphocytes, macrophages and dendritic cells $(10,11)$. TGF- $\beta 1$ is secreted as a latent complex that must be cleaved by proteases or reactive oxygen species (ROS) in order to become active (12). TGF- $\beta 1$ seems to be an important stimulant of growth arrest in many cell types, however in immunity, its predominant role is anti-inflammatory, inducing de-activation of T-lymphocytes and macrophages, thus promoting downregulation of humoral and cellular immune responses $(9,12-15)$. The above functions of TGF- $\beta 1$ seem to uncover its multifunctional role in tumor pathogenesis, acting either as a tumor growth inhibitor or as an enhancer of tumor progression via immune cell suppression. Although multiple mechanisms are involved in the control of TGF- 11 activity and its impact on tumor development and severity, transcriptional mechanisms regulated by various exogenous and endogenous factors are thought to be crucial. We have previously shown that TGF- $\$ 1$ mRNA levels are increased in cervical intraepithelial neoplastic tissues, especially in grade II lesions as compared to normal epithelia (1). According to the above observations other RT-PCR analysis-based studies demonstrate variations in TGF- $\beta 1$ transcript levels among CIN and normal cervical specimens $(7,8)$. Relevant reports on TGF- $\beta 1$ protein levels indicate decreased intracellular TGF- $\$ 1$ expression in neoplastic epithelium but increased extracellular levels in cervical cancer stroma (16). Additionally, other in vitro and in vivo observations on freshly isolated cervical carcinoma cells and cervicovaginal washings of patients with cervical cancer, respectively, have demonstrated local elevation of TGF- 31 , supporting the hypothesis that tumor progression may be indirectly promoted by TGF- $\beta 1$ via immunosuppression $(17,18)$.

The implication of VEGF in tumorigenesis has been well demonstrated. VEGF, as an endothelial cell-specific mitogen, induces cell proliferation and enhances vascular permeability, therefore promoting tumor development (19). Increased VEGF mRNA and protein expression has been associated with CIN and cervical cancer (1,4-6,20). VEGF expression levels in the uterine cervix have also been related with local tumor progression, its metastatic potential and poor prognosis $(5,6,20,21)$.

It has recently been shown that transcription factors known to be implicated in mammalian development and cell differentiation may also play a major role in the establishment and progression of several types of tumors. A novel member of this family of tumor-related transcriptional regulators is Yin-Yang 1 (YY1). YY1 is believed to be a constitutive factor, present in most cell types, affecting negatively or positively the transcription of a large number of viral and cellular genes including those involved in the control of cell growth, development, differentiation and tumor establishment (22-26). The putative role of YY1 in tumorigenesis is mainly supported by its known interaction with cell cycle regulation (27). YY1 overexpression is considered to be an important malignant marker, as it has been found in several tumor types including those of the prostate (28). Overexpression of YY1 may also regulate resistance and inhibit tumor cell destruction by the host immune system which may lead to tumor progression (27). The link between YY1 and GFs was established following reports indicating significant induction of YY1 expression in media rich in GFs, including IGF1 and FGF2 $(29,30)$. In HPVrelated cervical SIL and cancer, YY1 has been associated with repressing effects on HPV 16 and HPV 18 upstream regulatory region (URR) enhancer or promoter motifs which critically influence E6/E7 expression levels and thus the immortalizing and transforming potential of these strains in the cervix (31). The role of YY1 in cervical cancer and its precursor lesions however has not been investigated thus far.

Despite the established involvement of VEGF and TGF- 11 in the malignant conversion processes in different tissues including uterus cervix, the biological and prognostic significance of their expression variations between different grades of benign and cancerous tissues has not been well defined. The important issue that the present study addressed was how the gained knowledge from cervical tissue specimens, in terms of abnormal GF gene expression, could be similarly monitored in easily obtained Pap smear samples and whether the derived information could have clinical implications in prognosis management practices. Moreover, we investigated for the first time the implication of novel tumor-related transcriptional regulators, such as YY1, in cervical neoplasia and cancer, as well as the association of YY1 expression with exogenous factors known to confer to cervical malignant transformation, such as HPV infection.

\section{Materials and methods}

Study cases. Sixteen Pap smears randomly selected from patients diagnosed with cervical intraepithelial neoplasia and 6 relevant samples derived from individuals with cervical carcinomas were included in the study. A total of 19 Pap smear samples from histologically normal cervices served as the control group (Table I). All specimens were obtained during non-surgical ablation of Pap smear material for diagnostic purposes at the Department of Obstetrics and Gynecology of the University Hospital of Crete. Sections of samples were histologically evaluated to establish the diagnosis. The purity of the pathological samples was estimated by using H\&E staining to ensure that each sample contained $>80 \%$ neoplastic cells. Specimens were snap-frozen in liquid nitrogen at the time of ablation and stored at $-80^{\circ} \mathrm{C}$ until RNA extraction. Tumor smears included 5 squamous- and 1 adenocarcinoma, while the CIN group consisted of 6 lowgrade (CINI) and 10 high-grade (CINII and CINIII) squamous intraepithelial lesions (referred to as LG-SIL and HG-SIL respectively). The control group matched the 2 pathological groups (CIN, cancer) with respect to age (median, 45 years; range, 24-82 years) (Table I). Specimens' disease severity in addition with other clinical characteristics including age and HPV infection status, was correlated with the expression profiles of the studied genes in terms of their contribution to the altered patterns of mRNA expression between the various histological groups.

Additionally, paraffin sections of cervical tissues from 43 HPV-positive patients, including 16 LG-SIL, 11 HG-SIL, and 16 squamous cervical carcinomas, were subjected to YY1 immunohistochemistry analysis (Table I). All paraffin tissues were obtained from the Pathology Departments of the Hospital General No. 68 (HGZ) and the Hospital de Ginecologia y Obstetricia No. 3 CMN 'La Raza', Instituto Mexicano del 
Table I. The clinicopathological characteristics of the specimens.

\begin{tabular}{|c|c|c|c|c|}
\hline & Normal & LG-SIL & HG-SIL & Cancer \\
\hline \multicolumn{5}{|l|}{ Pap smears } \\
\hline No. of cases & 19 & 6 & 10 & 6 \\
\hline Median age & 45 & 35.3 & 42.2 & 55 \\
\hline $\begin{array}{l}\text { HPV status } \\
\text { HPV18 }(\%) \\
\text { HPV16 (\%) }\end{array}$ & $\begin{array}{c}3(15.7) \\
0\end{array}$ & $\begin{array}{c}3(50) \\
0\end{array}$ & $\begin{array}{l}5(50) \\
1(10)\end{array}$ & $\begin{array}{l}4(66.6) \\
2(33.3)\end{array}$ \\
\hline $\begin{array}{l}\text { VEGF } \\
\text { VEGF overexpression }(\%) \\
\text { Mean relative mRNA value } \pm \text { SEM }^{\mathrm{a}} \\
\text { Significance }(\mathrm{P})^{\mathrm{b}}\end{array}$ & & $\begin{array}{c}0 \\
0.52 \pm 0.1 \\
\text { LG/I }\end{array}$ & $\begin{array}{c}7(70) \\
3.67 \pm 0.8 \\
\text { LG/Ca: } 0 .\end{array}$ & $\begin{array}{l}5(83.3) \\
2.68 \pm 0.5 \\
\text { a: NS }\end{array}$ \\
\hline $\begin{array}{l}\text { TGF- } \beta 1 \\
\text { TGF- } \beta 1 \text { overexpression }(\%) \\
\text { Mean relative mRNA value } \pm \text { SEM } \\
\text { Significance }(\mathrm{P})^{\mathrm{b}}\end{array}$ & & $\begin{array}{r}0 \\
0.49 \pm 0.12 \\
\text { LG/ }\end{array}$ & $\begin{array}{c}7(70) \\
3.36 \pm 0.9 \\
\text { LG/Ca: NS }\end{array}$ & $\begin{array}{l}2(33.3) \\
2.7 \pm 0.8 \\
0.009\end{array}$ \\
\hline $\begin{array}{l}\text { YY1 } \\
\text { YY1 overexpression }(\%) \\
\text { Mean relative mRNA value } \pm \text { SEM } \\
\text { Significance }(\mathrm{P})^{\mathrm{b}}\end{array}$ & & $\begin{array}{r}5(83.3) \\
5.48 \pm 2 \\
\text { LC }\end{array}$ & $\begin{array}{c}9(90) \\
18.9 \pm 5.8 \\
\text { LG/Ca: NS }\end{array}$ & $\begin{array}{l}4(66.6) \\
15.52 \pm 11 \\
\mathrm{NS}\end{array}$ \\
\hline $\begin{array}{l}\text { Tissue specimens } \\
\text { No. of cases }\end{array}$ & & 16 & 11 & 16 \\
\hline $\begin{array}{l}\text { HPV status } \\
\text { HPV18 } \\
\text { HPV16 }\end{array}$ & & $\begin{array}{l}6 \\
8\end{array}$ & $\begin{array}{l}6 \\
5\end{array}$ & $\begin{array}{r}6 \\
10\end{array}$ \\
\hline
\end{tabular}

Seguro Social (IMSS) in Mexico City. HPV infection was confirmed and typed by PCR analysis as described below.

Histological cell types of the examined tumors and intraepithelial lesions were assigned according to the WHO Classification, whereas staging was reviewed based on the International Federation of Obstetrics and Gynecology (FIGO) staging system (32). None of the patients in the tumor groups had undergone any radio- or chemo-therapeutic treatment prior to the time of the sample collection, while the patients with CIN or normal individuals had not undergone any previous treatment of the cervix, including surgical or laser vaporization. Informed consent was obtained from all the individuals that participated in the study. The study protocol conformed to the ethical guidelines of the 1975 Declaration of Helsinki as reflected in a priori approval by the human research committees of the participating hospitals.

DNA extraction and HPV detection. Genomic and viral DNA was extracted from part of each smear or tissue sample by using the standard phenol/chloroform protocol (33). Amplification of HPV DNA in the studied specimens was originally performed by polymerase chain reaction (PCR) using pairs of primers specific for the E6 gene region of the HPV18 and HPV 16 viral strains. All samples were first examined for the presence of amplifiable DNA by using primers for the 32 -microglobulin gene and screened for the presence of non-type specific HPV DNA using the general HPV primers (HPVGP), $\mathrm{GP}^{+} / \mathrm{GP}^{+}$ (33). PCR reactions were performed in a total volume of $25 \mu 1$ containing $5 \mu \mathrm{M}$ of 10X PCR buffer [ $200 \mathrm{mM}$ Tris- $\mathrm{HCl}$ (pH 8.4), $500 \mathrm{mM} \mathrm{KCl}], 1.5 \mathrm{mM} \mathrm{MgCl}_{2}, 0.2 \mathrm{mM}$ of each deoxynucleotide (dNTPs), 0.6 units of recombinant Taq DNA polymerase (Invitrogen Ltd., UK) and $100 \mathrm{ng}$ of extracted DNA. All samples were subjected to 40 cycles of amplification as previously described (33). Primer sequences and concentrations used, as well as the expected PCR fragment sizes are summarized in Table I. Of each reaction mixture, $10 \mu \mathrm{l}$ was electrophoresed on $2 \%$ ethidium bromide-stained agarose gel and analysed for confirmation of the presence of the correct viral DNA amplimer. All positive samples were repeated twice for verification. Appropriate positive controls were included in each PCR reaction in order to establish the specificity of primer directed amplification. Recombinant plasmids carrying 
Table II. Primer sequences used for QRT-PCR.

\begin{tabular}{|c|c|c|c|c|}
\hline Primer pair & Sequence (5'-3') & $\begin{array}{l}\text { Concentration } \\
(\mathrm{nM})\end{array}$ & $\begin{array}{l}\text { Annealing temperature } \\
\qquad\left({ }^{\circ} \mathrm{C}\right)\end{array}$ & $\begin{array}{l}\text { Fragment size } \\
\qquad \text { (bp) }\end{array}$ \\
\hline HPVGP & $\begin{array}{l}\text { TTTGTTACTGTGGTAGATACTAC } \\
\text { GAAAAATAAACTGTAAATCATATTC }\end{array}$ & 1200 & 43 & 150 \\
\hline HPV18 & $\begin{array}{l}\text { AAACTAACTAACACTGGGTTATACA } \\
\text { ATGGCACTGGCCTCTATAGT }\end{array}$ & 500 & 56 & 143 \\
\hline HPV16 & $\begin{array}{l}\text { CTGCAAGCAACAGTTACTGCGACG } \\
\text { CATACATCGACCGGTCCACC }\end{array}$ & 500 & 56 & 315 \\
\hline B2-m & $\begin{array}{l}\text { TCCAACATCAACATCTTGGT } \\
\text { TCCCCCAAATTCTAAGCAGA }\end{array}$ & 400 & 56 & 123 \\
\hline VEGF & $\begin{array}{l}\text { ATGACGAGGGCCTGGAGTGTG } \\
\text { CCTATGTGCTGGCCTTGGTGAG }\end{array}$ & 300 & 60 & 91 \\
\hline TGF- $ß 1$ & $\begin{array}{l}\text { AAGGACCTCGGCTGGAAGTG } \\
\text { CCCGGGTTATGCTGGTTGTA }\end{array}$ & 400 & 60 & 137 \\
\hline YY1 & $\begin{array}{l}\text { GGAATACCTGGCATTGACC } \\
\text { GTGGTGGTGGTGGTCATGGTC }\end{array}$ & 300 & 58 & 127 \\
\hline GAPDH & $\begin{array}{l}\text { GGAAGGTGAAGGTCGGAGTCA } \\
\text { GTCATTGATGGCAACAATATCCACT }\end{array}$ & 300 & 60 & 101 \\
\hline
\end{tabular}

HPV-type specific sequences served as positive controls for HPV16 and HPV18 genome detection, whereas HeLa cells transfected with conserved L1 sequences among most HPV strains were used as a relevant control template for HPVGP amplification.

RNA extraction and cDNA synthesis. Total RNA was extracted from each specimen using the LS Trizol reagent (Invitrogen Ltd, UK) according to the manufacturer's instructions (1). RNA concentration and purity was determined on a UV/ VIS spectrophotometer (Hitachi Instruments Inc., USA) by evaluation of the A260/A280 ratios. RNA integrity was examined by denaturing polyacrylamide gel electrophoresis. Reverse transcription reactions for the preparation of firststrand cDNA were conducted in a total volume of $20 \mu \mathrm{l}$ by using the Promega RT system kit (Promega Corporation, USA). Briefly, for each sample $1 \mu \mathrm{g}$ of its RNA was preheated at $70^{\circ} \mathrm{C}$ for $10 \mathrm{~min}$ and then subjected to reverse transcription by adding the appropriate reagent mix supplemented by random hexamers as amplification primers. Each reaction mix was initially incubated at $42^{\circ} \mathrm{C}$ for $60 \mathrm{~min}$, followed by an additional incubation step at $95^{\circ} \mathrm{C}$ for 5 min mediating the complete inactivation of the reverse transcriptase. cDNAs were stored at $-20^{\circ} \mathrm{C}$ until use.

Quantitative real-time polymerase chain reaction ( $Q R T-P C R)$ analysis. Transcribed products were subjected to real-time PCR assay with $\mathrm{SYBR}^{\circledR}$-Green I in an Mx3000P programmable thermal controller apparatus (Stratagene). For each target gene $1 \mu 1$ of cDNA was amplified in a total volume of $20 \mu 1$ containing 2x Brilliant SYBR ${ }^{\circledR}$-Green QPCR Master mix (supplemented with $2.5 \mathrm{mM} \mathrm{MgCl}$ ), $30 \mathrm{mM}$ ROX as passive reference dye, and the appropriate concentration of each primer set. Glyceraldehyde-3-phosphate dehydrogenase (GAPDH) was used as internal control, in order to normalize target gene mRNA expression levels. All primer pairs were designed to span at least one intron in order to avoid amplification of contaminating genomic DNA along with cDNA. Primer sequences and their annealing conditions are listed in Table II. All reactions were performed in triplicate. Cervical samples were subjected to 45 cycles of amplification, comprised of denaturation at $95^{\circ} \mathrm{C}$ for $30 \mathrm{sec}$, annealing for $30 \mathrm{sec}$ at the appropriate temperature for each primer set tested, and elongation at $72^{\circ} \mathrm{C}$ for $30 \mathrm{sec}$. After amplification, standard curves were constructed, from samples used in a series of consecutive dilutions, for both the gene of interest and the internal control (GAPDH). Dissociation curves were also performed for all the tested genes to exclude the presence of byproducts or primer-dimer formation. Data were collected during annealing and extension with two measurements at each step and at all times during melting curve analysis. The correct PCR products were further confirmed by analysis on $2 \%$ ethidium bromide-stained agarose gels. For all tested samples the mRNA expression of both the target and the housekeeping gene (GAPDH) was calculated, projecting, with the help of the standard curve, the Ct value of all unknown samples to a relative mRNA quantity. Normalized mRNA values for each target gene were derived by dividing the relative mRNA value of each sample by the corresponding relative quantity of GAPDH mRNA.

Immunohistochemistry. Formalin-fixed paraffin-embedded cervical sections $(4 \mu \mathrm{m})$ were deparaffinized with heat and xylene treatment, and then re-hydrated with graded ethanol and distilled water. Antigen retrieval was performed by immersing the slides for $5 \mathrm{~min}$ into boiling $0.01 \%$ sodium 
A

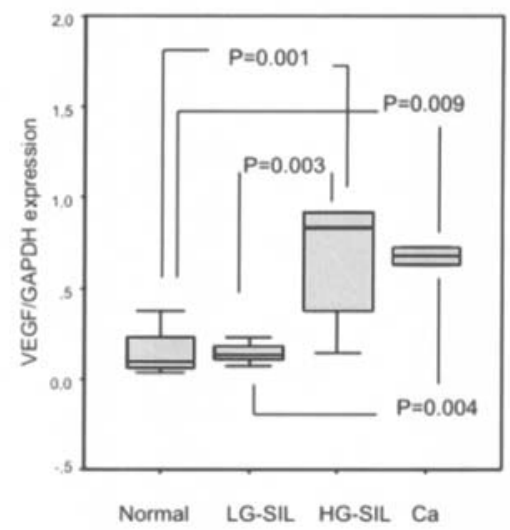

B

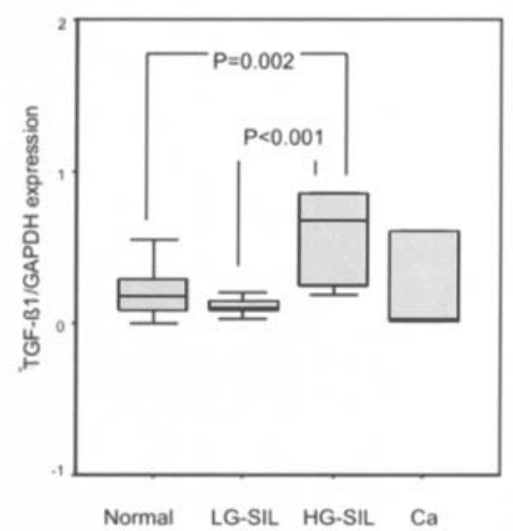

C

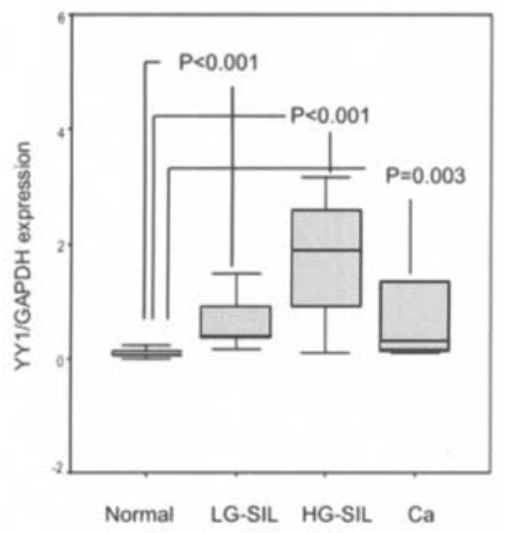

Figure 1. Box plots presenting the mean \pm SEM values of (A) VEGF, (B) TGF- 31 , and (C) YY1 mRNA expression (defined as ratios of target gene copies/GAPDH copies) assessed in Pap smears by QRT-PCR. All target genes were significantly overexpressed in LG-SIL and HG-SIL, while only VEGF and YY1 transcript levels were significantly higher in tumors compared to normal samples. The statistical significance of the expression differences among the various histological subgroups were determined using non-parametric pair-wise Mann-Whitney U test.

citrate. Endogenous peroxidase activity and unspecific antibody binding was reduced by treating the slides with $3 \%$ $\mathrm{H}_{2} \mathrm{O}_{2}$ in methanol for $25 \mathrm{~min}$, and $1 \% \mathrm{BSA}$ in phosphate buffer (PBS) $\mathrm{pH} 7.4$ for $60 \mathrm{~min}$, respectively. Rabbit polyclonal anti-human YY1 (Santa Cruz Biotechnology Inc., CA, USA) was used at $1 / 250$ dilution in PBS/BSA $0.1 \%$, as the primary antibody. The same concentration of rabbit $\operatorname{IgG}$ serum was used as negative control. Following overnight incubation with the primary antibody at room temperature, the slides were washed 5 times with PBS for 8 min each. In order to reduce the staining variability, all samples were processed at the same time in a single experiment using a single batch of antibody dilution. After washing, the sections were further incubated with an anti-rabbit biotinylated secondary antibody and a streptavidin-HRP conjugate (both provided by Universal LSAB kit, Dako Corporation, Carpinteria, CA, USA), according to the manufacturer's instructions. Color visualization was established by staining the slides with 3,3'diaminobenzidine tetrahydrochloride (DAB) (Dako) for 1 to $5 \mathrm{~min}$ in the dark. Color development was arrested with distilled water and the slides were counterstained with hematoxylin. Finally, sections were dehydrated in graded ethanol baths (70,90 and 100\%) and xylene and protected with mounting medium. Levels of YY1 protein expression were scored under a light microscope at x400 magnification. Score estimation was based on the evaluation of the mean signal intensity (scale 0 to $4+$ ) by two independent specialists.

Statistical analysis. VEGF, TGF-ß1 and YY1 mRNA expression was compared among normal, LG-SIL, HG-SIL, and cancer groups and correlated with their HPV status. The Kolmogorov-Smirnov goodness of fit test was initially used to determine whether the expression follows a normal distribution pattern. Where appropriate non-parametric tests (KruskalWallis $\mathrm{H}$ and Mann-Whitney U tests) or Chi-square $\left(\chi^{2}\right)$ Pearson analysis, were applied for evaluation of statistical significance. The comparison of YY1 staining score among different histological groups, as well as the association of mRNA expression patterns with clinical parameters, including age and HPV infection status, was assessed similarly. Data are presented as the mean \pm standard error of the mean (mean \pm SEM). Spearman's rank correlation was used to evaluate the significance of the given target gene mRNA co-expression pair-wise between the indicated groups. Probability values (pvalues $)<0.05$ were considered statistically significant; while all statistical analyses were 2-sided. Statistical analyses were performed using the SPSS 11.5 software (SPSS, Chicago, IL).

\section{Results}

$V E G F$ and TGF- $\beta 1$ overexpression correlates with progression of low-grade to high-grade cervical intraepithelial neoplasia; constitutive up-regulation of YY1 transcript levels in CIN and cervical cancer. Two approaches were applied for the comparative evaluation of the gene expression among the indicated groups. According to the first approach, the normalized values of CIN and cervical cancer samples were divided by the mean normalized value of all normal controls. The derived ratio represents the relative expression of each CIN or tumor sample to normal controls. Two-fold increase (a value $\geq 2$ ) or decrease (a value $\leq 0.5$ ) was considered as target gene mRNA overexpression or underexpression, respectively. Alternatively, the second approach was based on the comparison of the mean normalized expression values for each target gene among all the four subgroups studied (normal, LG-SIL, HG-SIL, cancer).

The results based on the analysis by the first approach are presented in Table I. The percentage of HG-SIL samples with VEGF or TGF-ß1 overexpression was significantly higher when compared to LG-SIL specimens. The majority of cancer samples also showed statistically significant VEGF overexpression compared to that observed in LG-SIL cases, while for TGF- 31 a significant underexpression following the progression of HG-SIL to cancer was observed in $66.6 \%$ of the cancer samples. YY1 overexpression patterns were detected in all pathological groups, however, the mean relative YY1 mRNA expression in HG-SIL was marginally higher $(\mathrm{p}=0.061)$ compared to LG-SIL. 
A

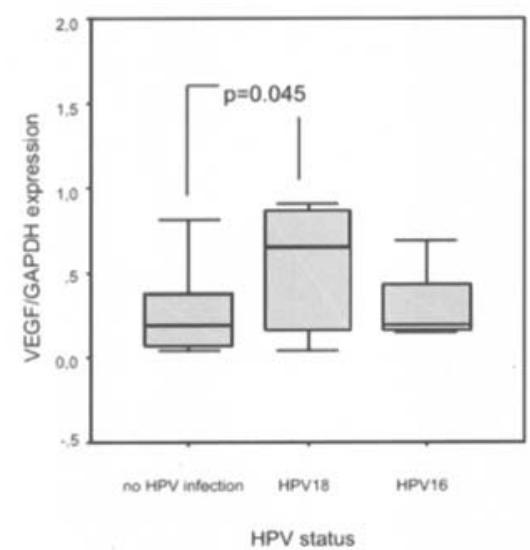

B

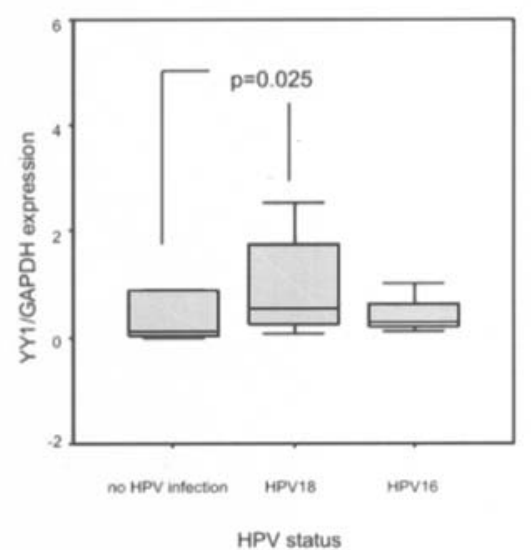

C

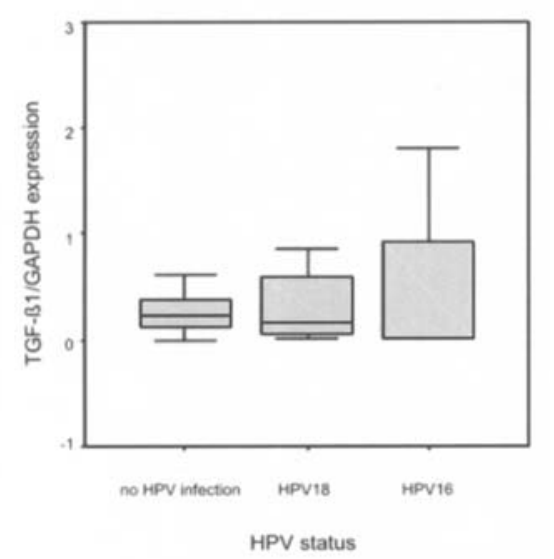

Figure 2. Association patterns of (A) VEGF, (B) YY1, and (C) TGF-ß1 mRNA expression with the HPV status of the specimens. HPV18 infection was found to be significantly correlated with YY1 and VEGF overexpression. Statistical significance was assessed by non-parametric Mann-Whitney U test.

Since the above statistical estimations were based on differences in absolute numbers and percentages of samples presenting a certain expression status as defined above, it was reasonable that the fluctuation of the expression values among the studied samples was not considered as an evaluation parameter. Therefore, the objective of the second approach for evaluation of the results was a comparative assessment of the differences in mean normalized gene expression values defined among the studied subgroups. According to this approach, all genes studied showed statistically significant expression variations among the groups of normal, LG-SIL, HG-SIL and cancer (Kruskal Wallis, $\mathrm{p}=0.001, \mathrm{p}=0.005$, $\mathrm{p}<0.0001$ for VEGF, TGF-ß1 and YY1 respectively) (Fig. 1). Specifically, VEGF expression in normal samples was significantly decreased compared to that observed in HG-SIL and cancer samples (Fig. 1A). Disrupted VEGF expression profiles were also established between LG-SIL and HG-SIL as well as in cancer specimens indicating a progressive elevation of VEGF mRNA levels upon cervical neoplastic transformation to cancer. With respect to TGF-B1, HG-SIL samples showed elevated gene transcript levels compared to normal and LG-SIL cases, as similarly observed with VEGF (Fig. 1B). However, there was no detectable significant association between the TGF- 11 mRNA patterns detected in the cancer group and those detected in the other subgroups. The YY1 expression patterns showed multiple variations among the studied categories, with the controls presenting basal transcript levels (Fig. 1C). Following the onset of an initial cervical intraepithelial neoplasia (LG-SIL) and the progression to HG-SIL and cancer, YY1 mRNA was significantly increased. However, YY1 up-regulation in HG-SIL was only marginally significant compared to LG-SIL ( $\mathrm{p}=0.062)$ or cancer specimens $(\mathrm{p}=0.055)$.

Co-expression of VEGF and TGF- $\beta 1$ mRNAs in normal cervix and LG-SIL; YYI expression correlates negatively with VEGF and TGF- $\beta 1$ transcript levels upon the onset of the cervical neoplastic transformation. Spearman rank correlation was performed in order to test the co-expression patterns regarding the mRNA levels of the examined genes in a pair-wise manner in normal, LG-SIL, HG-SIL and cancer samples. The test is applied for examination of the proportional (positive) or conversely proportional (negative) correlation of the expression patterns of two gene products. The results are summarized in Table III. Based on the findings, in normal cervical samples VEGF and TGF- 11 showed a co-expression status (Spearman's rho, $\mathrm{p}=0.002$ ), while no correlation was established between YY1 and VEGF or TGF-ß1 (Table IIIA). The positive correlation between VEGF and TGF- 31 was retained in LG-SIL specimens (Spearman's rho, $\mathrm{p}=0.005$ ), however, new negative correlations that were not present in the control group were formed upon the onset of the cervical neoplastic transformation. Specifically, in the LG-SIL group VEGF and YY1, as well as TGF- 31 and YY1, were found to be expressed in a reverse manner (Spearman's rho, $\mathrm{p}<0.001$ and $\mathrm{p}=0.005$, respectively) (Table IIIB). In contrast, in HG-SIL and cancer groups there were no correlations demonstrated between the examined expression patterns, indicating that all types of correlations present in controls and LG lesions had been lost (Table IIIC and D). From the above results, we deduce that the co-expression profiles of all studied gene products differ significantly mainly among normal, LG-SIL and HGSIL cervical specimens. Those findings suggest that the interplay between VEGF, TGF- 31 and YY1 plays a crucial role in the onset and progression of the cervical neoplastic conversion process.

Implication of YY1 and VEGF mRNA overexpression in HPV infection of the uterine cervix. Non-parametric tests (KruskalWallis $\mathrm{H}$ and Mann-Whitney U tests) or Chi-square $\left(\chi^{2}\right)$ Pearson analysis, were applied where appropriate for evaluation of the contribution of various clinical parameters, including the patient age and HPV infection status, to the altered patterns of mRNA expression among the different histological groups. All specimens were initially tested for HPV genome detection, followed by typing of HPV18 and HPV16 strains in HPVpositive cases, as previously described (33) (Table I). A statistically significant overall difference in the presence of HPV DNA was established among normal, CIN and tumor groups ( $\mathrm{p}=0.042)$. The majority of the CIN samples $(56.25 \%)$ was infected by the virus, with dominance of the HPV 18 
Table III. Spearman's correlation rho and p-values in (A) normal, (B) LG-SIL, (C) HG-SIL, and (D) cancer groups of patients.

\begin{tabular}{|c|c|c|c|c|c|}
\hline A & & & VEGFN & TGF-ß1N & YY1N \\
\hline \multirow[t]{8}{*}{ Spearman's rho } & VEGFN & $\begin{array}{l}\text { Correlation coefficient } \\
\text { Significance (2-tailed) }\end{array}$ & 1.000 & & \\
\hline & & $\mathrm{n}$ & 19 & & \\
\hline & TGF-ß1N & Correlation coefficient & 0.675 & 1.000 & \\
\hline & & Significance (2-tailed) & 0.002 & & \\
\hline & & $\mathrm{n}$ & 19 & 19 & \\
\hline & YY1N & Correlation coefficient & 0.329 & 0.154 & 1.000 \\
\hline & & Significance (2-tailed) & 0.170 & 0.529 & \\
\hline & & $\mathrm{n}$ & 19 & 19 & 19 \\
\hline B & & & VEGFLG & TGF-ß1LG & YY1LG \\
\hline \multirow[t]{8}{*}{ Spearman's rho } & VEGFLG & $\begin{array}{l}\text { Correlation coefficient } \\
\text { Significance (2-tailed) }\end{array}$ & 1.000 & & \\
\hline & & $\mathrm{n}$ & 6 & & \\
\hline & TGF-ß1LG & Correlation coefficient & 0.943 & 1.000 & \\
\hline & & Significance (2-tailed) & 0.005 & & \\
\hline & & $\mathrm{n}$ & 6 & 6 & \\
\hline & YY1LG & Correlation coefficient & -1.000 & -0.943 & 1.000 \\
\hline & & Significance (2-tailed) & 0.000 & 0.005 & \\
\hline & & $\mathrm{n}$ & 6 & 6 & 6 \\
\hline $\mathrm{C}$ & & & VEGFHG & TGF-ß1HG & YY1HG \\
\hline \multirow[t]{8}{*}{ Spearman's rho } & VEGFHG & $\begin{array}{l}\text { Correlation coefficient } \\
\text { Significance (2-tailed) }\end{array}$ & 1.000 & & \\
\hline & & $\mathrm{n}$ & 10 & & \\
\hline & TGF-ß1HG & Correlation coefficient & 0.115 & 1.000 & \\
\hline & & Significance (2-tailed) & 0.751 & & \\
\hline & & $\mathrm{n}$ & 10 & 10 & \\
\hline & YY1HG & Correlation coefficient & -0.079 & -0.152 & 1.000 \\
\hline & & Significance (2-tailed) & 0.829 & 0.676 & \\
\hline & & $\mathrm{n}$ & 10 & 10 & 10 \\
\hline $\mathrm{D}$ & & & VEGFCa & TGF-ß1Ca & YY1Ca \\
\hline \multirow[t]{8}{*}{ Spearman's rho } & VEGFCa & $\begin{array}{l}\text { Correlation coefficient } \\
\text { Significance (2-tailed) }\end{array}$ & 1.000 & & \\
\hline & & $\mathrm{n}$ & 6 & & \\
\hline & TGF- $ß 1 \mathrm{Ca}$ & Correlation coefficient & 0.029 & 1.000 & \\
\hline & & Significance (2-tailed) & 0.957 & & \\
\hline & & $\mathrm{n}$ & 6 & 6 & \\
\hline & YY1Ca & Correlation coefficient & 0.371 & 0.486 & 1.000 \\
\hline & & Significance (2-tailed) & 0.468 & 0.329 & \\
\hline & & $\mathrm{n}$ & 6 & 6 & 6 \\
\hline
\end{tabular}

subtype (50\% of samples), while all tumor specimens were either HPV18- (66.6\%) or HPV16- (33.3\%) positive. Our findings from HPV typing are consistent with previously reported data on the incidence of HPV18 and HPV16 strains in pathological cervical tissues and Pap smears collected from the same geographical region $(34,35)$. Examination for the presence of mRNA expression variations between infected and non-infected cases revealed that VEGF and YY1 mRNA 


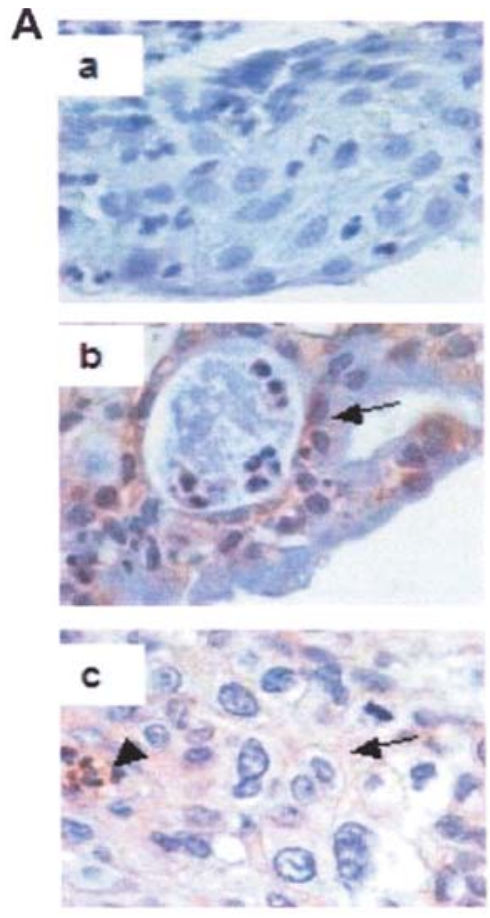

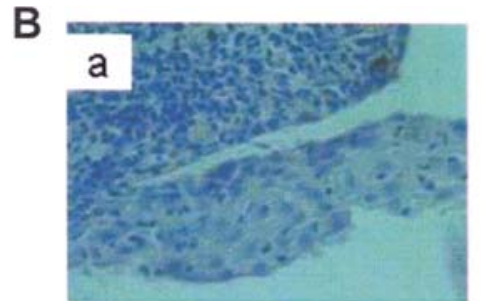
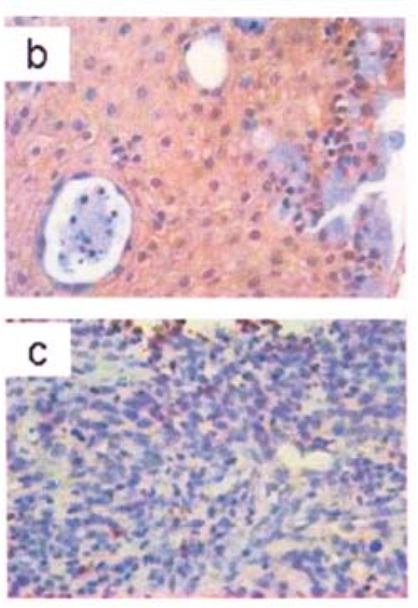

C

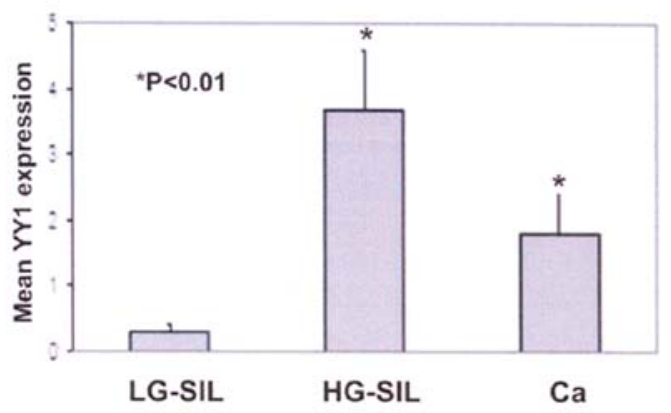

D

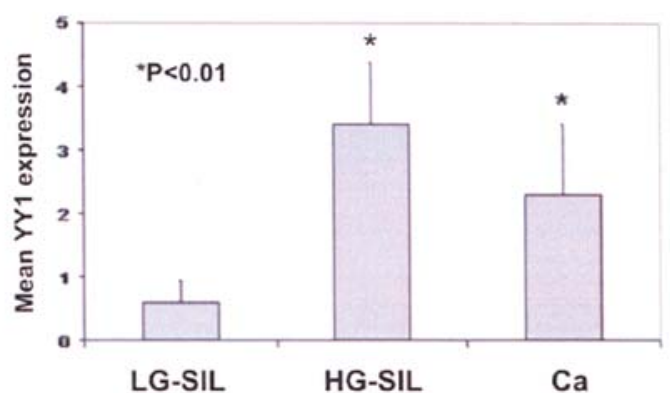

Figure 3. Representative YY1 protein expression in (A) HPV16- and (B) HPV18-positive cervical sections with different histology, as assessed by immunohistochemistry. The analysis showed (a) basal YY1 expression in LG-SIL samples, (b) clear cytoplasmic staining in HG-SIL (indicated by the arrow), (c) weak staining in tumor cells of the Ca group (arrow), while strong cytoplasmic and nuclear expression was observed in inflammatory cells of the same group (arrow head). Magnification: x100. (C and D) Quantitative analysis of YY1 expression between LG-SIL, HG-SIL and cancer specimens in (C) HPV16or (D) HPV18-infected specimens. Each bar represents the mean signal intensity evaluated by the total number of slides in each group (Table I). Each slide was scored by examination of 5 representative spots. *p-value: LG-SIL vs HG-SIL or cancer. Statistical analysis was performed using the Kruskal-Wallis H test.

expression in the HPV18-DNA positive samples was significantly higher compared to HPV18-DNA negative samples (Fig. 2A and B). However, we did not establish any significant differences in the above transcript levels among infected and non-infected LG-SIL, HG-SIL and tumor specimens. Similarly, we did not detect any association between infection and TGF-ß1 mRNA expression (Fig. 2C).

The evaluation of age contribution in the expression profiles of target genes revealed that only in the cancer group the patients with TGF- $\beta 1$ overexpression were younger compared to those with TGF- 31 downregulation (45 \pm 7.5 vs. $67 \pm 5.972$ years), (Kruskal-Wallis H test, $\mathrm{p}=0.064$ ). All other associations tested, including those regarding VEGF and YY1, were not statistically significant.

Increased YY1 protein levels are associated with HG-SIL, cervical cancer and HPV infection of the uterine cervix. In order to test whether the YY1 protein expression correlates with the results obtained by RT-PCR analysis, we examined the distribution and abundance of YY1 protein in cervical sections with different histology. Thus, 16 LG-SIL, 11 HGSIL and 16 cervical tumor specimens, all derived from HPVinfected patients, were subjected to immunohistochemistry analysis for YY1 protein expression. The distribution of HPV18 and HPV 16 strains among the above groups is summarized in Table I. Virus detection and typing was confirmed by PCR as described in Materials and methods.

For both HPV groups, HPV 16+ (Fig. 3A and C) and $\mathrm{HPV}^{+} 8^{+}$(Fig. 3B and D), significant variations in YY1 protein expression were observed among LG-SIL, HG-SIL and cancer samples, as similarly observed with YY1 mRNAs. LG-SIL presented basal levels of YY1 protein (1+) (Fig. 3Aa and $\mathrm{Ba})$ which were significantly lower compared to those observed in HG-SIL samples $(3+)(p=0.0001)$ (Fig. 3Ab, Bb, $\mathrm{C}$ and D). YY1 staining was heterogeneously distributed in the cells of HG-SIL origin, whereas in LG-SIL the staining was preferentially present in the basal epithelial cells. Although cancer samples showed a strong cytoplasmic and nuclear YY1 staining in inflammatory polymorphonuclear cells $(\mathrm{PMN})$ and particularly in neutrophils $(3+, 4+)$, the expression of YY1 in tumor cells was lower and limited only to the cytoplasm (2+) (Fig. 3Ac and Bc). Compared to HGSIL, the reduction in YY1 expression levels observed in cancer samples was also statistically significant $(\mathrm{p}=0.001)$ (Fig. 3C and D). The above findings indicate that the HPV infection of the uterine cervix by the most oncogenic HPV strains HPV18 and HPV16 not only consists of a negative background of cervical intraepithelial lesions and cervical cancer development, but it also correlates with YY1 overexpression.

\section{Discussion}

Our incomplete understanding of the molecular mechanisms involved in cervical carcinogenesis, as well as the absence of early prognostic factors, may account for the lack of optimal treatment and poor outcome in many cases. The identification of genome-wide changes in gene expression profiles among histologically different cervical groups is thought to be crucial in the prediction of the clinical course of the cervical pathology. 
In this study we provide novel evidence for the implication of YY1 in the malignant transformation of the uterine cervix and its association with HPV infection. Growth factors, such as VEGF and TGF- 31 with established involvement in uterus cervix carcinogenesis (1), were also tested for disrupted mRNA profiles during disease progression as well as for co-expression with YY1.

Our findings demonstrate significantly disrupted mRNA expression for all the examined genes among the different cervical subgroups tested. VEGF transcript levels were found to be significantly higher in HG-SIL and cancer histological types compared to controls, as we had previously observed in cervical tissues (1). Additionally, new correlations that failed to be established in tissue samples appeared; HG-SIL and cancer groups showed VEGF overexpression compared to LG-SIL. Both approaches applied for the evaluation of VEGF expression variations among the examined subgroups showed that elevated VEGF levels are essential not only for the onset of the intraepithelial lesions but also for the progression of LG-SIL to HG-SIL and finally to cancer. Our data are in accordance with previous reports indicating increased VEGF mRNA levels in both CIN and invasive tumors of the uterine cervix that have been further associated with increased microvessel density $(4,36)$. VEGF is thought to be undoubtedly one of the most important promoters of angiogenesis, and it is overexpressed in a variety of human tumors thereby contributing significantly to tumor growth and metastasis $(37,38)$. Consequently, these findings suggest that VEGF could also be a significant mediator of angiogenesis in CIN and cervical carcinomas presenting overexpression patterns proportional to the severity of the cervical lesion.

Similarly, TGF- $1 \mathrm{mRNA}$ expression was found to be significantly elevated in high-grade lesions derived from either cervical tissues or Pap smears compared to the normal group. Moreover, in both cervical materials and independently of the applied evaluation method, LG-SIL presented reduced TGF- 11 mRNA expression compared to HG-SIL, as was observed with VEGF. Despite the fact that the TGF- $\beta 1$ relative expression detected in HG-SIL and cervical cancer samples revealed a TGF- $\beta 1$ underexpression in cancer, the evaluation of the mean normalized TGF- $\beta 1$ expression values between the two groups did not result in any significant association. The above observation suggests that the disrupted VEGF and TGF- $\beta 1$ patterns present in HG-SIL possibly trigger a commitment of neoplastic cells to an advanced transformation process, leading finally to malignancy. The dual role of TGF$\beta 1$ as suppressor or enhancer of tumor progression has been well demonstrated $(39,40)$. However, little is yet known about how TGF-ß1-induced transcriptional control regulates cell proliferation and differentiation in uterine cervix. In the literature there are conflicting reports indicating variations in TGF- $\beta 1$ mRNA or protein levels between CIN and normal tissue specimens $(7,8,41)$. The observed overexpression of TGF-ß1 mRNA in HG-SIL compared to normal or LG-SIL specimens suggests a role of immunosuppression in the progression of cervical neoplasia. The positive correlation between VEGF and TGF-ß1 expression in the groups of normal and LG-SIL was further established by Spearman's rank correlation analysis. However, we observed loss of the indicated co-expression in HG-SIL and tumor groups, possibly reflecting a rather random disruption in GF expression in cases with high disease severity.

The involvement of YY1 in tumorigenesis is supported by recent observations indicating association of elevated YY1 protein levels with different types of human benign lesions and tumors in various tissues including prostate (28), brain and B-cell lymphomas (unpublished data). However, no reports are available regarding the expression of YY1 in CIN and cancer. According to our findings, YY1 appears to confer to malignant transformation of the uterine cervix, since its protein expression was significantly differentiated during disease development. Higher and lower YY1 levels were observed in HG- and LG-SIL respectively, while tumor cells presented intermediate staining, although significantly reduced compared to HG-SIL. This could be explained by the fact that YY1 may act as a transcriptional repressor of some gene promoters implicated in tumor development, thus reduced YY1 levels could be essential for tumor establishment. Despite that both YY1 protein and mRNA expression patterns followed the same trend among the different pathological groups, YY1 mRNA overexpression in HG-SIL compared to LG-SIL or cancer specimens was marginally significant. This discrepancy could be attributed most likely to difficulties faced in the statistical evaluation of the results due to the small number of LG-SIL and tumor samples. However, we cannot rule out activation of post-transcriptional mechanisms in addition to well-known post-translational modifications in YY1 protein (22). Overall, the findings suggest that YY1 becomes and remains constitutively elevated during the progression of cervical neoplasia, which could be potentially considered as an indicator of disease development. Interestingly, the gene co-expression analysis performed in our study revealed a negative correlation between YY1 and TGF- 11 mRNA transcript levels in LG-SIL, which is lost in advanced disease stages. It has been previously reported that YY1 may act as a transcriptional activator of TGF- $\beta 1$ expression via direct binding to the TGF- $\beta$ promoter (42). However, the efficiency of this binding appeared to be positively influenced by the presence of certain YY1 DNA binding site-related polymorphisms and the YY1 protein availability (43). In contrast, it has been shown that the interaction of YY1 with intracellular signaling effectors of TGF- $\beta 1$, such as Smad proteins, is able to inhibit TGF- $\beta$-induced pathways $(23,44)$. Hence, we can postulate that via different mechanisms low YY1 expression (although increased compared to normal tissue) may act as an inhibitor of TGF-ß1-related signaling pathways, therefore promoting the onset of neoplastic transformation, whereas significantly elevated YY1 levels may confer TGF- $\beta 1$ activation and disease progression. The above assumption is consistent with our findings, since both gene transcripts were increased in HG-SIL compared to controls and LG-SIL, but only YY1 mRNA levels were elevated in LG-SIL (compared to controls).

Increased YY1 transcript and protein levels were also associated with the presence of HPV18 or HPV16 infection. YY1 has been reported to be involved in the regulation of HPV18 promoter activity. Multiple YY1 binding sites have been identified in the HPV18 upstream regulatory region (URR), of which the promoter-proximal site plays a functional role (22). While the majority of the HPV 18 cancer isolates 
carry mutations or deletions in YY1 binding sites which confer transcriptional activation of the promoter (45-48), the significance of the promoter proximal YY1 site as a conditional activating site has been recently reported in HeLa cells. When another sequence $130 \mathrm{bp} 5$ ' upstream relative to the YY1 site termed the 'switch region' is present in the HPV18 URR, the promoter proximal YY1 site serves as a positive promoter regulatory element. This suggests a functional interaction between YY1 and the 'switch' region via binding to $\mathrm{C} / \mathrm{EBP} B$ protein, which may determine the transcriptional activity of YY1 (22). Therefore, in the context of the intact HPV18 URR, YY1 is thought to be an activator of HPV18 transcription in HeLa cells. The present findings provide evidence that overexpression of YY1 may promote the immortalizing and transforming potential of HPV that consequently results in progression of benign cervical lesions to pre-malignant or malignant conditions. YY1 binding sites have also been detected in the promoters of E6 and E7 oncoproteins of HPV16 suppressing the HPV-specific long control region (LCR) $(22,49)$. Deletions or mutations in the above sites have been reported that may also be associated with malignant conversion in the absence of virus integration $(31,49)$. The above statements are consistent with our observations of high YY1 protein expression in HPV16- and HPV18-infected HG-SIL and cancer tissue specimens. HPVinfected PMN cells present in cancer sections showed the strongest cytoplasmic and nuclear YY1 staining among the examined cells, which possibly reflects the crucial role of YY1 in the survival and proliferation of infiltrated infected cells. The variation in the incidence of different HPV subtypes between the sample collections derived from Greece and Mexico could be attributed to preferential geographical distribution of certain viral strains $(34,35)$. Although the observed frequency of HPV18 and HPV16 in HG-SIL and tumor Pap smears is consistent with previously reported data derived from the same geographical region $(34,35)$, we cannot exclude that the lack of detection of a higher percentage of HPV18-positive HG-SIL smears could be related to the clinical method performed for sample collection, and thus to low undetectable viral load present in those samples.

In summary, our findings support the role of VEGF and TGF- $\$ 1$ mRNA disruption in the malignant transformation of the uterine cervix and introduce YY1 overexpression as a new candidate with potential prognostic significance in cervical disease development. Moreover, the identification of VEGF, TGF- 11 and YY1 transcript patterns in Pap smears could provide easily obtained information for the aggressiveness and the biological evaluation of the cervical neoplasias without the necessity of surgically removed cervical tissue. However, further research with a large cohort of samples is necessary to elucidate the prognostic and therapeutic value of the above findings.

\section{References}

1. Soufla G, Sifakis S, Baritaki S, Zafiropoulos A, Koumantakis E and Spandidos DA: VEGF, FGF2, TGFß1 and TGFßR1 mRNA expression levels correlate with the malignant transformation of the uterine cervix. Cancer Lett 221: 105-118, 2005.

2. Smith-McCune KK and Weidner N: Demonstration and characterization of the angiogenic properties of cervical dysplasia. Cancer Res 54: 800-804, 1994.
3. Munger K and Phelps WC: The human papillomavirus E7 protein as a transforming and transactivating factor. Biochim Biophy Acta 1155: 111-123, 1993.

4. Guidi AJ, Abu-Jawdeh G, Berse B, Jackman RW, Tognazzi K, Dvorak HF and Brown LF: Vascular permeability factor (vascular endothelial growth factor) expression and angiogenesis in cervical neoplasia. J Natl Cancer Inst 87: 1237-1245, 1995.

5. Cheng WF, Chen CA, Lee CN, Wei LH, Hsieh FJ and Hsieh CY: Vascular endothelial growth factor and prognosis of cervical carcinoma. Obstet Gynecol 96: 721-726, 2000.

6. Lee IJ, Park KR, Lee KK, et al: Prognostic value of vascular endothelial growth factor in Stage IB carcinoma of the uterine cervix. Int J Radiat Oncol Biol Phys 54: 768-779, 2002.

7. El-Sherif AM, Seth R, Tighe PJ and Jenkins D: Decreased synthesis and expression of TGF-beta1, beta2, and beta3 in epithelium of HPV 16-positive cervical precancer: a study by microdissection, quantitative RT-PCR, and immunocytochemistry. J Pathol 192: 494-501, 2000.

8. Giannini SL, Al-Saleh W, Piron H, et al: Cytokine expression in squamous intraepithelial lesions of the uterine cervix: implications for the generation of local immunosuppression. Clin Exp Immunol 113: 183-189, 1998.

9. McCartney-Francis NL and Wahl SM: Transforming growth factor beta: a matter of life and death. J Leukoc Biol 55: 401-409, 1994.

10. Shalaby MR and Ammann AJ: Suppression of immune cell function in vitro by recombinant human transforming growth factor-beta. Cell Immunol 112: 343-350, 1988.

11. Aragane Y, Riemann H, Bhardwaj RS, et al: IL-12 is expressed and released by human keratinocytes and epidermoid carcinoma cell lines. J Immunol 153: 5366-5372, 1994.

12. Blobe GC, Schiemann WP and Lodish HF: Role of transforming growth factor beta in human disease. N Engl J Med 342: 1350-1358, 2000.

13. Hobbs K, Negri J, Klinnert M, Rosenwasser LJ and Borish L: Interleukin-10 and transforming growth factor-beta promoter polymorphisms in allergies and asthma. Am J Respir Crit Care Med 158: 1958-1962, 1998.

14. Tsunawaki S, Sporn M, Ding A and Nathan C: Deactivation of macrophages by transforming growth factor-beta. Nature 334 : 260-262, 1988 .

15. Letterio JJ and Roberts AB: Regulation of immune responses by TGF-beta. Annu Rev Immunol 16: 137-161, 1998.

16. Comerci JT, Runowicz CD, Flanders KC, et al: Altered expression of transforming growth factor-beta 1 in cervical neoplasia as an early biomarker in carcinogenesis of the uterine cervix. Cancer 77: 1107-1114, 1996.

17. Santin AD, Hermonat PL, Hiserodt JC, et al: Differential transforming growth factor-beta secretion in adenocarcinoma and squamous cell carcinoma of the uterine cervix. Gynecol Oncol 64: 477-480, 1997.

18. Tjiong MY, van der Vange N, ter Schegget JS, Burger MP and ten Kate TA: Out, Cytokines in cervicovaginal washing fluid from patients with cervical neoplasia. Cytokine 14: 357-360, 2001.

19. Bergers $\mathrm{G}$ and Benjamin LE: Tumorigenesis and the angiogenic switch. Nat Rev Cancer 3: 401-410, 2003.

20. Cheng WF, Chen CA, Lee CN, Chen TM, Hsieh FJ and Hsieh CY: Vascular endothelial growth factor in cervical carcinoma. Obstet Gynecol 93: 761-765, 1999.

21. Tjalma W, Weyler J, Weyn B, et al: The association between vascular endothelial growth factor, microvessel density and clinicopathological features in invasive cervical cancer. Eur $\mathbf{J}$ Obstet Gynecol RB 92: 251-257, 2000.

22. Shi Y, Lee JS and Galvin KM: Everything you have ever wanted to know about Yin Yang 1. Biochem Biophys Acta 1332: F49-F66, 1997.

23. Thomas MJ and Seto E: Unlocking the mechanisms of transcription factor YY1: are chromatin modifying enzymes the key? Gene 236: 197-208, 1999.

24. Krippner-Heidenreich A, Walsemann G, Beyrouthy MJ, et al: Caspase-dependent regulation and subcellular redistribution of the transcriptional modulator YY1 during apoptosis. Mol Cell Biol 25: 3704-3714, 2005.

25. Austen M, Luscher B and Luscher-Firzlaff JM: Characterization of the transcriptional regulator YY1. The bipartite transactivation domain is independent of interaction with the TATA box-binding protein, transcription factor IIB, TAFII55, or cAMP-responsive element-binding protein (CPB)-binding protein. J Biol Chem 272: 1709-1717, 1997. 
26. Donohoe ME, Zhang X, McGinnis L, Biggers J, Li E and Shi Y: Targeted disruption of mouse Yin Yang 1 transcription factor results in peri-implantation lethality. Mol Cell Biol 19: 7237-7244, 1999.

27. Gordon S, Akopyan G, Garban H and Bonavida B: Transcription factor YY1: structure, function, and therapeutic implications in cancer biology. Oncogene 25: 1125-1142, 2006.

28. Seligson D, Horvath S, Huerta-Yepez S, et al: Expression of transcription factor Yin Yang 1 in prostate cancer. Int J Oncol 27: 131-141, 2005.

29. Flanagan JR: Autologous stimulation of YY1 transcription factor expression: role of an insulin-like growth factor. Cell Growth Differ 2: 185-190, 1995.

30. Santiago FS, Lowe HC, Bobryshev YV and Khachigian LM: Induction of the transcriptional repressor Yin Yang-1 by vascular cell injury. Autocrine/paracrine role of endogenous fibroblast growth factor-2. J Biol Chem 276: 41143-41149, 2001.

31. Dong XP, Stubenrauch F, Beyer-Finkler E and Pfister H: Prevalence of deletions of YY1-binding sites in episomal HPV 16 DNA from cervical cancers. Int J Cancer 58: 803-808, 1994.

32. Benedet JL, Bender H, Jones H III, Ngan HY and Pecorelli S: FIGO staging classifications and clinical practice guidelines in the management of gynecologic cancers, FIGO Committee on Gynecologic Oncology. Int J Gynaecol Obstet 70: 209-262, 2000.

33. Lyronis ID, Baritaki S, Bizakis I, Tsardi M and Spandidos DA: Evaluation of the prevalence of human papillomavirus and Epstein-Barr virus in esophageal squamous cell carcinomas. Int J Biol Markers 20: 5-10, 2005.

34. Dokianakis DN, Sourvinos G, Sakkas S, Athanasiadou E and Spandidos DA: Detection of HPV and ras gene mutations in cervical smears from female genital lesions. Oncol Rep 5: 1195-1198, 1998.

35. Dokianakis DN, Papaefthimiou M, Tsiveleka A and Spandidos DA: High prevalence of HPV18 in correlation with ras gene mutations and clinicopathological parameters in cervical cancer studied from stained cytological smears. Oncol Rep 6: 1327-1331, 1999.

36. Obermair A, Bancher-Todesca D, Bilgi S, Kaider A, Kohlberger P and Mullauer-Ertl S: Correlation of vascular endothelial growth factor expression and microvessel density in cervical intraepithelial neoplasia. J Natl Cancer Inst 89: 1212-1217, 1997.
37. Ferrara N: Vascular endothelial growth factor: basic science and clinical progress. Endocr Rev 25: 581-611, 2004.

38. Abulafia O, Triest WE and Sherer DM: Angiogenesis in malignancies of the female genital tract. Gynecol Oncol 72: 220-231, 1999.

39. Derynck R, Akhurst RJ and Balmain A: TGF-beta signaling in tumor suppression and cancer progression. Nat Genet 29: 117-129, 2001.

40. Moustakas A, Pardali K, Gaal A and Heldin CH: Mechanisms of TGF-beta signaling in regulation of cell growth and differentiation. Immunol Lett 82: 85-91, 2002.

41. Hazelbag S, Fleuren GJ, Baelde JJ, Schuuring E, Kenter GG and Gorter A: Cytokine profile of cervical cancer cells. Gynecol Oncol 83: 235-243, 2001.

42. Silverman ES, Palmer LJ, Subramaniam V, et al: Transforming growth factor-betal promoter polymorphism C-509T is associated with asthma. Am J Respir Crit Care Med 169: 214-219, 2004.

43. Grainger DJ, Heathcote K, Chiano M, et al: Genetic control of the circulating concentration of transforming growth factor type beta1. Hum Mol Genet 8: 93-97, 1999.

44. Kurisaki K, Kurisaki A, Valcourt U, et al: Nuclear factor YY1 inhibits transforming growth factor beta- and bone morphogenetic protein-induced cell differentiation. Mol Cell Biol 23: 4494-4510, 2003.

45. Romanczuk H, Villa LL and Schlegel R: The viral transcriptional regulatory region upstream of the E6 and E7 genes is a major determinant of the differential immortalization activities of human papillomavirus types 16 and 18. J Virol 65: 2739-2744, 1991.

46. Rose B, Steger G, Dong XP, et al: Point mutations in SP1 motifs in the upstream regulatory region of human papillomavirus type 18 isolates from cervical cancers increase promoter activity. J Gen Virol 79: 1659-1663, 1998.

47. Villa LL and Schlegel R: Differences in transformation activity between HPV18 and HPV16 map to the viral LCR-E6-E7 region. Virology 181: 374-377, 1991.

48. Hoppe-Seyler F and Butz K: Cellular control of human papillomavirus oncogene transcription. Mol Carcinog 10: 134-141, 1994.

49. Dong XP and Pfister H: Overlapping YY1- and aberrant SP1binding sites proximal to the early promoter of human papillomavirus type 16. J Gen Virol 80: 2097-2101, 1999. 